This is a self-archived - parallel published version of this article in the publication archive of the University of Vaasa. It might differ from the original.

\title{
Predicting cryptocurrency defaults
}

Author(s): Grobys, Klaus; Sapkota, Niranjan

Title: $\quad$ Predicting cryptocurrency defaults

Year: $\quad 2020$

Version: Accepted manuscript

Copyright (C) 2020 Taylor \& Francis Group. This is an Accepted Manuscript of an article published by Taylor \& Francis in Applied Economics Letters on o3 May 2020, available online:

http://www.tandfonline.com/10.1080/o0036846.2020.1752903

Please cite the original version:

Grobys, K. \& Sapkota, N. (2020). Predicting cryptocurrency defaults. Applied Economics 52(46), 5060-5076. https://doi.org/10.1080/00036846.2020.1752903 


\title{
Predicting Cryptocurrency Defaults
}

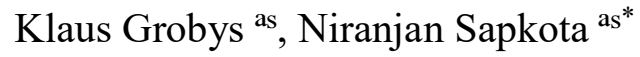 \\ ${ }^{a}$ University of Vaasa, School of Accounting and Finance
}

\begin{abstract}
We examine all available 146 Proof-of-Work based cryptocurrencies that started trading prior to the end of 2014 and track their performance until December 2018 . We find that about $60 \%$ of those cryptocurrencies were eventually in default. The substantial sums of money involved mean those bankruptcies will have an enormous societal impact. Employing cryptocurrencyspecific data, we estimate a model based on linear discriminant analysis to predict such defaults. Our model is capable of explaining $87 \%$ of cryptocurrency bankruptcies after only one month of trading and could serve as a screening tool for investors keen to boost overall portfolio performance and avoid investing in unreliable cryptocurrencies.
\end{abstract}

\section{JEL Classification: G12, G14}

Key Words: Cryptocurrency, Bitcoin, Bankruptcy, Default, Credit risk

\author{
* Correspondence to: \\ K. Grobys (A. Prof. of Finance) Department of Accounting and Finance, University of Vaasa, Wolffintie 34, \\ 65200 Vaasa, Finland \\ E-mail: klaus.grobys@uva.fi
}

N. Sapkota (Ph.D. Student in Finance) Department of Accounting and Finance, University of Vaasa, Wolffintie 34, 65200 Vaasa, Finland

Email: niranjan.sapkota@uva.fi

\footnotetext{
${ }^{\mathrm{s}}$ We would like to thank Simon Moore for a lively, interesting, and detailed discussion of our paper in the U.S. Business Magazine Forbes entitled "How To Tell If Your Cryptocurrency Will Go Bust" on May 28, 2019 . Moreover, valuable comments were received from participants of the 2019 Graduate School Seminar at the University of Vaasa. We also received valuable comments from the 2019 Aalto University Graduate School of Finance Workshop at the University of Jyväskyla. In particular, we would like to thank Mika Veihekoski for an encouraging discussion and helpful comments. Furthermore, we are grateful for having received valuable comments from the 2019 Economics \& Finance Seminar at Hanken School of Economics. Especially we would like to thank Timo Korkeamäki, Kenneth Högholm and Emilia Vähämaa for useful comments. Moreover, we received interesting comments from the participants from the 2019 Blockchain Seminar at the University of Vaasa. We would like to thank Robert Faff and the participants of the FINANCE, PROPERTY, TECHNOLOGY, AND THE ECONOMY CONFERENCE 2019, University of South Australia. Finally, we would like to thank an anonymous reviewer for helpful comments.
} 


\section{Introduction}

Facing the zeitgeist of digitalization, Bill Gates stated that "the future of money is digital currency." Since the advent of Bitcoin - the first cryptocurrency traded - the number of cryptocurrencies has increased exponentially and there are now over 2,000 cryptocurrencies traded on over 16,000 markets around the world. The main advantages of cryptocurrencies are transparency and 24-hour accessibility. Transactions of cryptocurrencies are all recorded on the open public ledger called the blockchain. This decentralized mechanism gives cryptocurrencies an unparalleled transparency. The technology behind the blockchain is revolutionary, but understanding it is challenging, especially for people without a technical background.

In contrast to traditional investments, cryptocurrencies carry different risks. For instance, Rauchs and Hileman (2017) reports that the chance of cryptocurrency exchanges being hacked is $74-79 \%$. Taking the legal perspective, Kethineni and Cao (2019) argue that cryptocurrencies became the currency of choice for many drug dealers and extortionists because of the opportunities to hide behind the presumed privacy and anonymity. Maume (2019), who explores Initial Coin Offerings (ICO), highlights that the potential lack of regulation and enforcement is particularly tempting for scammers and other miscreants. In contrast to traditional currency markets, cryptocurrency markets also involve credit risk: As a stylized fact, among all the cryptocurrencies launched prior to December 31 2014, 59\% went in default by the end of 2018, and the reasons for defaults are manifold. ${ }^{2}$

As of February 2019, the overall market capitalization in the digital asset market is more than USD 120 billion with Bitcoin dominating slightly with more than $50 \%{ }^{3}$ In this regard, Fry and Cheah (2016, p.350) highlight that "from an economic perspective the sums of money involved are substantial," and accordingly, the societal impact of losses due to defaults in the digital asset market may be enormous. Howell, Niessner and Yermack (2019, p.1) define three types of digital assets which are often referred to as coins. Specifically, the first type of digital asset is defined as a general-purpose medium of exchange and store of value cryptocurrency, such as Bitcoin. The second type of digital asset is a security token, which represents a conventional security that is recorded and exchanged on a blockchain to

\footnotetext{
${ }^{1}$ The Bloomberg interview took place on October 2, 2014.

2 The dead coin tracking website coinopsy.com lists the following as the main reasons for default: abandoned, abandoned/website, abandoned/volume, abandoned/buyback, abandoned/scam, scam, scam project/virus, joke, no exchanges/struggling, failed fork, failed/pre-mine no/low trade volume, pump and dump, and crashed (see https://www.coinopsy.com/dead-coins/).

${ }^{3}$ See https://coinmarketcap.com (accessed on 15 February 2019, 11:00 EST).
} 
reduce transaction costs and create a record of ownership, whereas the third type of digital assets is a utility token, which gives its holder consumptive rights to access a product or service. In Tables 1 and 2, we provide a demographic overview of new and bankrupt cryptocurrencies in different years.

Table 1. Population of cryptocurrencies including tokens

\begin{tabular}{|c|c|c|c|c|c|c|c|c|}
\hline Year & $\begin{array}{c}\text { Before } \\
\text { Apr 28, 2013 } \\
\end{array}$ & $\begin{array}{c}\text { Mar- Dec } \\
2013 \\
\end{array}$ & 2014 & 2015 & 2016 & 2017 & 2018 & 2019 \\
\hline $\begin{array}{l}\text { New } \\
\text { cryptocurrencies }\end{array}$ & 7 & 60 & 452 & 207 & 298 & 800 & 1187 & 168 \\
\hline $\begin{array}{l}\text { Default } \\
\text { cryptocurrencies }\end{array}$ & 0 & 0 & 2 & 150 & 363 & 213 & 743 & 307 \\
\hline $\begin{array}{l}\text { Total } \\
\text { cryptocurrencies }\end{array}$ & 7 & 67 & 517 & 577 & 663 & 1353 & 2073 & 2147 \\
\hline
\end{tabular}

Note: This table reports the numbers of new, bankrupt, and total cryptocurrencies during each year from April 2013 till April 2019. It is generated using the historical snapshot available at coinmarketcap.com.

Table 2. Life Span of default cryptocurrencies including tokens

\begin{tabular}{|c|c|c|c|c|c|c|c|c|}
\hline & Default Year & 2014 & 2015 & 2016 & 2017 & 2018 & $\begin{array}{c}\text { Jan-Apr } \\
2019 \\
\end{array}$ & Total \\
\hline \multicolumn{9}{|c|}{ Number of default cryptocurrencies } \\
\hline \multirow{8}{*}{ 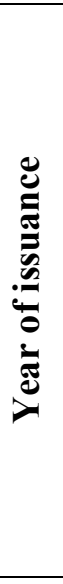 } & $\begin{array}{l}\text { Before Apr 28, } \\
2013\end{array}$ & 0 & 0 & 0 & 0 & 1 & 0 & 1 \\
\hline & Mar-Dec 2013 & 2 & 1 & 17 & 3 & 11 & 4 & 38 \\
\hline & 2014 & & 149 & 133 & 34 & 58 & 13 & $387^{*}$ \\
\hline & 2015 & & & 213 & 56 & 98 & 20 & 387 \\
\hline & 2016 & & & & 120 & 174 & 26 & 320 \\
\hline & 2017 & & & & & 401 & 72 & 473 \\
\hline & 2018 & & & & & & 172 & 172 \\
\hline & $\begin{array}{l}\text { Total number of } \\
\text { default } \\
\text { cryptocurrencies }\end{array}$ & 2 & 150 & 363 & 213 & 743 & 307 & 1778 \\
\hline
\end{tabular}

Note: This table reports the numbers of bankrupt cryptocurrencies with their specific year of issuance and year of bankruptcy. It is generated using the historical snapshot available at coinmarketcap.com.

*It includes all cryptocurrencies and tokens using different consensus mechanisms. Out of which 86 cryptocurrencies that we used for our analysis are based on PoW consensus protocols. 
Figure 1 shows that the numbers of default cryptocurrencies are increasing in comparison to the new cryptocurrencies added to the digital finance world after 2018 . Specifically, we find that as of April 2019 there are altogether 1778 defaulted coins, however, 2147 coins are still in the digital asset market. ${ }^{4}$

Fig. 1. Demography of cryptocurrencies (Apr 2013 - Apr 2019)

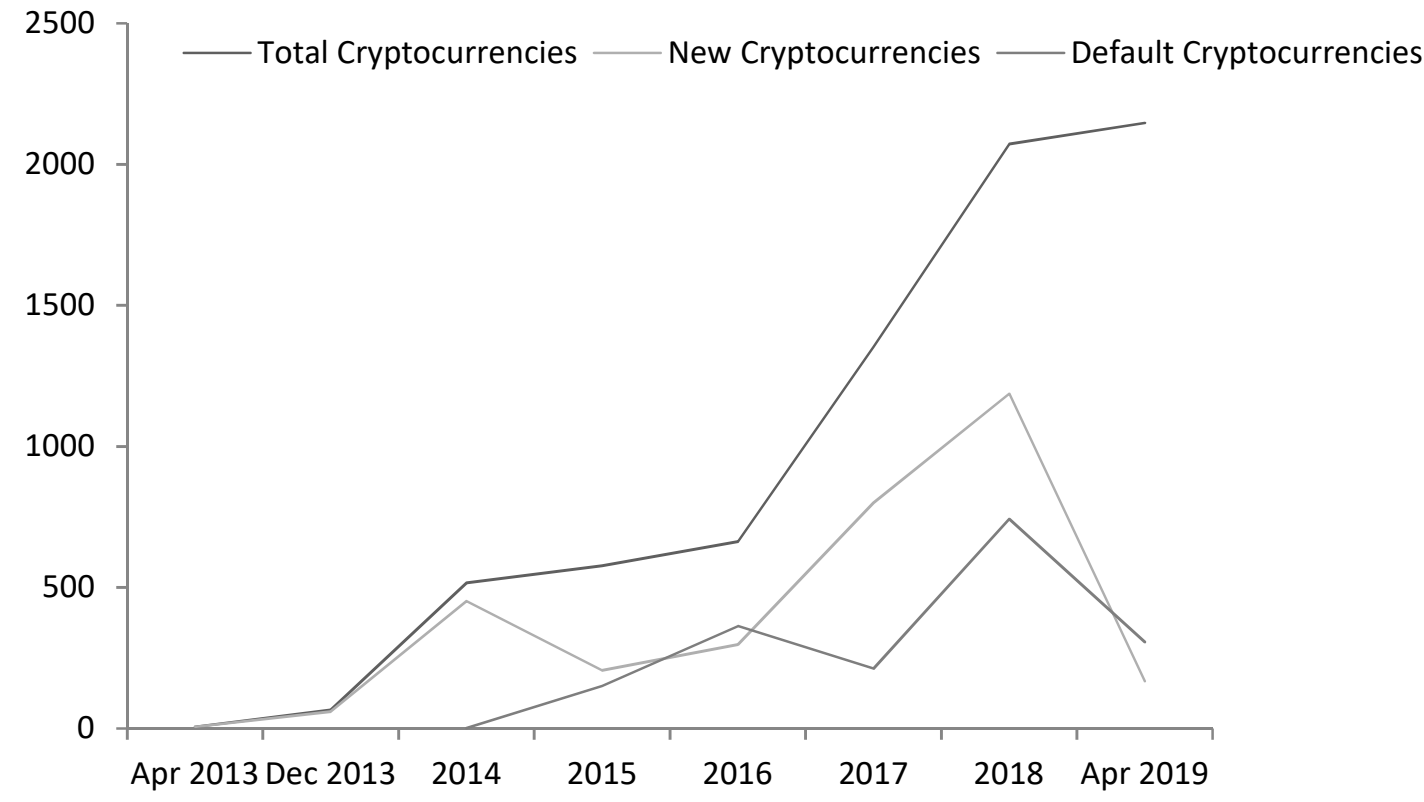

Note: This figure shows the evolutions of new, default and total cryptocurrencies. Cryptocurrencies correspond to all three types of digital assets as defined in Howell, Niessner and Yermack $(2019$, p.1) where the first type is defined as a general-purpose medium of exchange and store of value cryptocurrency, such as Bitcoin. The second type of cryptocurrencies is a security token, which represents a conventional security that is recorded and exchanged on a blockchain to reduce transaction costs and create a record of ownership, whereas the third type of cryptocurrencies is a utility token, which gives its holder consumptive rights to access a product or service.

It should not be surprising that in a zero-interest regime even the asset management industry pays ever more attention to digital assets as an investment alternative. Given the likelihood of digital assets ending up in default, it is surprising that there is no paper available exploring the extent to which a default of a digital asset is forecastable. This current paper fills this important gap in the new age of digital finance literature.

\footnotetext{
${ }^{4}$ Note that Howell, Niessner and Yermack (2019, p.1) define three types of coins, Figure 1 accounts for the whole universe of digital assets. For instance, as of 2014, 146 out of 517 coins were cryptocurrencies that have the Proof-of-Work consensus protocol which are subject of examination in this study.
} 
In our paper, we exclusively focus on the first category of digital asset defined as cryptocurrencies. As this type of digital asset is considered general-purpose medium of exchange, it is an alternative to traditional currency. We start our analysis by exploring which cryptocurrency-specific variables are accessible to the naïve investor. As we are interested in forecasting potential cryptocurrency defaults at an early stage, we focus on variables that are a part of the information set of the investor at most one month after a cryptocurrency started trading. Accordingly, we downloaded data for all cryptocurrencies launched before 2015 and followed those cryptocurrencies until the end of 2018. ${ }^{5}$ Specifically, our data set consists of 146 cryptocurrencies, of which 86 went bankrupt before the end of 2018 . We divided our dataset into two subsamples: The first subsample contains data on those cryptocurrencies that went into default and the second subsample contains the data of those cryptocurrencies that functioned until the end of our sample period. To analyze which of our variables have discriminative power, we then test which of the mean differences of our cryptocurrencyspecific variables for our two subsamples were statistically significant. We made use of those variables that exhibited significant differences in sample means in a multiple linear discriminant model. We compared the estimated bankruptcies with the actual numbers. Moreover, we applied bootstrapping techniques to investigate the robustness of our model involving Type-I and Type-II errors.

Our paper contributes to the new strand of digital finance literature exploring cryptocurrencies. Recent literature investigates the volatility of cryptocurrencies (Katisiampa, 2017; Balcilar, Bouri, Gupta, and Roubaud, 2017; Osterrieder and Lorenz, 2017; Ardia, Bluteau, and Rüede, 2018; Baur and Dimpfl, 2018; Borri, 2019), price spillovers between cryptocurrencies (Fry and Cheah, 2016), predictability of cryptocurrency time series (Catania, Grassi and Ravazzolo, 2019; Lahmiri and Bekiros, 2019; Omane-Adjepong, Alagidede and Akosah, 2019; Shen, Urquhart, and Wang, 2019), cryptocurrencies as investment assets (Urquhart 2016; Dyhrberg, 2016; Dwyer, 2015), and speculative bubbles in the cryptocurrency market (Cheah and Fry, 2015; Chaim and Laurini, 2019; Li, Tao, Su, and Lobont, 2019). Even though empirical evidence shows that the majority of cryptocurrencies

\footnotetext{
${ }^{5}$ It is also noteworthy that cryptocurrencies exhibit different types of consensus protocols to verify transactions such as Proof-of-Work, Proof-of-Stake or a mixture of both which is often referred to as Hybrid. Before 2015, however, there were only few cryptocurrencies issued that were implemented using the Proof of Stake (PoS) mechanism. PoS was first introduced by Sunny King and Scott Nadal in 2012 and later in 2013 Sunny King created the first cryptocurrency Peercoin (PPC) implementing the PoS protocol. PoS is created to solve the high energy consumption problem of Bitcoin which uses the Proof-of-Work mechanism. In order to keep our sample homogenous, we exclude those cryptocurrencies using a PoS mechanism from our sample.
} 
go into default, there is no paper available on the predictability of such cryptocurrency bankruptcy. Being able to forecast potential cryptocurrency defaults is important because the sums of money involved are substantial (Fry and Cheah, 2016). This paper fills this important gap in the literature while also complementing the large body of literature exploring the predictability of commercial bankruptcy. The publication of Altman's (1968) z-score model for predicting bankruptcy among manufacturing firms in the U.S.A, led to a wealth of research (Satish and Janakiram, 2011; Wang and Campbell, 2010; Lugovskaya, 2010), and Altman (2018) has recently provided an excellent overview of the relevant literature.

Moreover, Cheah and Fry (2015) and Osterrieder and Lorenz (2017) express concern that academic research on cryptocurrency is often focused on the legality of cryptocurrencies (Kethineni and Cao, 2019; Maume, 2019) rather than offering a comprehensive analysis of their statistical or financial aspects. Therefore, our paper contributes to the finance literature by adding a new perspective, credit risk. Finally, and from a more practical point of view, our paper also supports the finance industry by proposing a model that could be used for investment decisions. For instance, new digital asset management could use our model to determine which cryptocurrencies should be treated with caution owing to a high probability of default.

The results of this research show that bankruptcies among cryptocurrencies are predictable. Specifically, our model shows that we can predict 75 out of 86 cryptocurrency defaults. Employing 5000 bootstrap replications shows that the confidence interval for the point estimate indicating default does not overlap with the point estimate for the Type-I error. This shows that the discriminative power of our model is significant. Our results are in line with the literature on predicting firm bankruptcy (Altman, 1968, 1983, 2000, 2002; Altman, Haldeman, and Narayanan, 1977; Altman, Hartzell and Peck, 1995; Lugovskaya, 2010). Surprisingly, our model is not suitable for predicting the fate of functioning cryptocurrencies unlike Altman's (1968) z-score model or Altman, Haldeman, and Narayanan's (1977) ZETA model. We strongly encourage future research to elaborate on this issue.

The paper is organized as follows: The next section presents the empirical framework, including the model setup and robustness checks and the last section concludes. 


\section{Empirical framework}

\subsection{Multiple Linear Discriminant Analysis}

Our analysis is supported by data from the various sources. ${ }^{6}$ Each cryptocurrency has certain characteristics related to its history, specification, trading activities, reward, privacy, and scaling among others. Table A.1 in the appendix shows the categorized specification details of cryptocurrencies. We downloaded all cryptocurrencies that incorporated the Proof-ofWork $(\mathrm{PoW})^{7}$ mechanism and started trading between 2010 and the end of 2014 and considered a data period of four years ahead. ${ }^{8}$ In total, we retrieved 146 cryptocurrencies, of which 86 went into default in the sample period and 60 continued functioning. We define a cryptocurrency as being in a 'default state' when the cryptocurrency stopped trading, that is, there is no more evidence of any trading. ${ }^{9}$ Altman (2010, pp.4-5) emphasizes the importance of ratio analysis as an empirical tool in assessing the performance of business enterprises. Identifying variables that exhibit discriminative power is ultimately an empirical question. Therefore, the first step in our analysis was to explore variables that potentially discriminate between cryptocurrencies that ended up in default and those that remained functioning. Moreover, we wanted to account for variables that only the investor has access to at most one month after starting trading that support a decision on whether to invest in the relevant cryptocurrency at an early stage. Table A.2 in the appendix records 20 cryptocurrencyspecific variables that exhibit information that could be utilized. Unfortunately, some information was not available for some now defunct cryptocurrencies.

There are many cryptocurrencies that are pre-mined before being offered to the public. Pre-mining has some advantages like rewarding the developers or creating a balanced distribution of coins (e.g., units of a cryptocurrency) between developers and traders. However, a larger number of pre-mined coins could be a negative indication, as when the developer has a large percentage of available coins and could therefore opt to leverage the

\footnotetext{
${ }^{6} \mathrm{We}$ used the following sources: mapofcoins.com (name of cryptocurrency, categorization of 'running' and 'defunct'), coinmarketcap.com (historical price data), deadcoins.com (confirmation of categorization as 'defunct'), coinopsy.com/dead-coins (life span and founder information of dead coins), bitcointalk.org (announcement date and other technical specifications), and personal websites of coins for gathering any missing data.

${ }^{7} \mathrm{PoW}$ is the very first consensus algorithm in decentralized public blockchain where miners solve complex cryptographic puzzles to add a block to the blockchain in exchange for coin as rewards.

${ }^{8}$ We downloaded price history from the coinmarketcap.com. The earliest data provided by this website starts on 28 April 2013. Though Bitcoin (BTC), Litecoin (LTC), Namecoin (NMC), Terracoin (TRC), Devcoin (DVC) and, Novacoin (NVC) started trading before this date. To have uniformity and consistency across our data set, however, we set 28 April 2013 as the first day of trade for the above mentioned coins.

${ }^{9}$ There are a few cryptocurrencies in the list of functioning cryptocurrencies in coinmarketcap.com even though these cryptocurrencies do not exhibit any trading activities. In our data set, we adjusted for these errors.
} 
price before selling quickly. Cryptocurrencies exhibiting higher levels of pre-mining are under constant attack and carry a high manipulation risk $^{10}$. Therefore, investors are generally concerned about whether a particular cryptocurrency is pre-mined or not (which we account for by using a simple binary dummy variable), and also the fractions of pre-mined coins (it measures the extent to which the developers retain control over that particular cryptocurrency if the total coins are mined as the Pre-mined-to-Total-Coins-Ratio (PMTTCR)). Moreover, we accounted for block time, Day-1 return, Week-1 return, and Month-1 return after the respective cryptocurrency started trading. For instance, a positive return in the initial trading period could indicate the popularity of a particular cryptocurrency. We also compounded the corresponding time-congruent volatilities (Day-1, Week-1, and Month-1) simply as the corresponding squared return. Instead of interpreting each variable in isolation, our variables should be considered in the respective context. For instance, a slightly negative first day trading return with a low volatility in association with a high monthly volatility could indicate that the cryptocurrency did not attract attention following the announcement owing to a lack of social promotion, but the cryptocurrency could be subject to excessive speculation within the first month after trading. Generally, assets that are subject to excessive speculation may end up in trouble — or in default — at a later stage. Furthermore, reward per block shows the level of coin supply during that particular block interval. We include Minimum-Reward-ToTotal-Coin-Ratio (MTTCR) as a common comparative tool to measure the minimum level of controlled supply among the cryptocurrencies in our sample. Our model includes both an individual and a comparative level of minimum controlled supply. Finally, we also coded dummy variables for identifying both the cryptocurrency-specific algorithm and whether the cryptocurrency has a known founder. ${ }^{11}$

We report the descriptive statistics of our selected variables in Table A.3 in the appendix. Moreover, Table 3 reflects the variable means and the results of testing the difference in means for significance. We used a simple two-sample $t$-test to test the difference in sample means (Snedecor and Cochran, 1989). The sample differences of minimum reward, Day-1 and Month-1 returns, Day-1 volatility, and PMTTCR are statistically significant on at least a 5\% level (see Table 3). Moreover, the Month-1 volatility is at least marginally significant on a $10 \%$ level. Interestingly, we also find that among functioning cryptocurrencies, $58 \%$ of the founders remain anonymous, whereas among bankrupt

10 See https:/cryptodaily.co.uk/2018/08/premined-coins-like-xrp-trx-xlm-and-neo-are-causing-problems-forindex-funds (published on August 29, 2018).

${ }^{11}$ We categorized algorithms into three types; 'SHA' (Secure Hash Algorithm), 'Scrypt', and 'others'. 'Others' contains all other algorithms besides SHA and Scrypt family algorithms. 
cryptocurrencies that figure rises to $79 \%$. For a $95 \%$ confidence interval, the critical values for the binary-distributed variable known founder in the sample of functioning cryptocurrencies is between 0.50 and 0.66 , implying that the sample of bankrupt cryptocurrencies exhibits a significantly higher probability of the founder being anonymous, given a 5\% significance level. Moreover, for a 95\% confidence interval, the critical value for the binary-distributed variable scrypt algorithm in the sample of functioning cryptocurrencies is between 0.52 and 0.68 . As the sample average in the default sample is 0.80 , we can reject the null hypothesis that the sample means are equal, implying that those cryptocurrencies that ended up in default exhibit this specific algorithm more frequently.

More precisely, the definitions of our variables are as following:

Ret_D $1_{t}=\frac{\left(\text { Day }{\text { Close })_{t}-(\text { Day }}_{1} \text { Open }\right)_{t}}{\left(\text { Day } \text { Open }_{t}\right.}$,

where $\operatorname{Ret}_{-} D 1_{t}$ denotes the first day's return of cryptocurrency $t,\left(\text { Day } y_{1} C l o s e\right)_{t}$ denotes the first day's closing price of cryptocurrency $t$, and $\left(\text { Day } y_{1} O p e n\right)_{t}$ denotes the first day's opening price of cryptocurrency $t$.

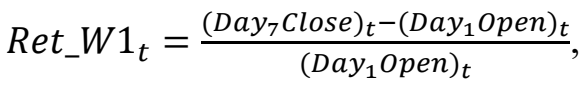

where $R e t \_W 1_{t}$ denotes the first week's return of cryptocurrency $t,\left(\text { Day }{ }_{7} C l o s e\right)_{t}$ denotes the closing price after the seventh day of cryptocurrency $t$, and $\left(\text { Day }_{1} O p e n\right)_{t}$ denotes the first day's opening price of the cryptocurrency $t$.

Ret $M 1_{t}=\frac{\left(\text { Day }_{30} \text { Close }\right)_{t}-\left(\text { Day }_{1} \text { Open }\right)_{t}}{\left(\text { Day }_{1} \text { Open }\right)_{t}}$,

where $\operatorname{Ret}_{-} M 1_{t}$ denotes the first month's return of the cryptocurrency $t$, $\left(\operatorname{Day}_{30} \mathrm{Close}\right)_{t}$ denotes the closing price of cryptocurrency $t$ after 30 trading days, and (Day $\left.{ }_{1} O p e n\right)_{t}$ denotes the first day's opening price of cryptocurrency $t$.

Vol_D $1_{t}=\left(\operatorname{Ret}_{-} D 1_{t}\right)^{2}$,

where $V o l_{-} D 1_{t}$ denotes the first day's volatility of cryptocurrency $t$, and $\operatorname{Ret}_{-} D 1_{t}$ denotes the first day's return of cryptocurrency $t$. 
$V o l \_W 1_{t}=\left(R e t \_W 1_{t}\right)^{2}$,

where $V o l_{-} W 1_{t}$ denotes the first week's volatility of cryptocurrency $t$, and $R e t_{-} W 1_{t}$ denotes the first week's return of cryptocurrency $t$.

Vol_M1 $1_{t}=\left(\operatorname{Ret}_{-} M 1_{t}\right)^{2}$,

where $V o l_{-} M 1_{t}$ denotes the first month's volatility of cryptocurrency $t$, and $\operatorname{Ret}_{-} M 1_{t}$ denotes the first month's return of cryptocurrency $t$.

Moreover, PMTTCR (Pre-Mined-To-Total-Coins-Ratio) indicates the fraction of coins that are allocated to the developers in relation to the total coins in circulation, given that a cryptocurrency is fully mined. (Note that developers with a large portion of coins in stake can manipulate the market with a so-called pump-and-dump strategy. Note also that if a large proportion of a cryptocurrency is pre-mined, this cryptocurrency could be subject to potential scam.) Further,

PMTTCR $_{t}=\frac{(\text { NUMBER OF PRE-MINED COINS })_{t}}{(\text { TOTAL COINS WHEN FULLY MINED })_{t}}$,

where $P M T T C R_{t}$ denotes the Pre-Mined-To-Total-Coins-Ratio of cryptocurrency $t$, $(N U M B E R \text { OF PRE - MINED COINS })_{t}$ denotes the number of pre-mined coins of cryptocurrency $t$, and (TOTAL COINS WHEN FULLY MINED) $t$ denotes the number of total coins of cryptocurrency $t$ when being fully mined.

The number of coins received by miners as a reward per block for any cryptocurrency shows how new coins are generated after every block time interval (which, in turn, varies among cryptocurrencies). Specifically, block time is the time it takes to verify one block. This also indicates how frequently the new coins are generated to reward the miners for verifying the block. Moreover, the coins rewarded for the miners are the new coins supplied to the market. Due to the limited supply of coins (at least for the majority of cryptocurrencies), the reward decreases over time. Minimum reward measures the lowest number of coins as a reward given to the miners. The mining of a cryptocurrency continues only if the rewards cover the mining cost. If the minimum reward is meager such that the mining cost cannot be 
covered, miners will stop mining and eventually that cryptocurrency is likely to end up in default. Therefore, minimum reward may be an important factor to consider in our current research's context. On the other hand, MTTCR (Minimum-Reward-to-Total-Coins-Ratio) measures the minimum level of controlled supply until the cryptocurrency is fully mined. Both, too much or too little supply of coins are not beneficial for the crypto economy. Further,

$M_{T T C R}=\frac{(\text { MINIMUM REWARDS PER BLOCK EXCLUDING BONUS REWARDS })_{t}}{(\text { TOTAL COINS WHEN FULLY MINED })_{t}}$,

where $M T T C R_{t}$ denotes the Minimum-Reward-to-Total-Coins-Ratio of cryptocurrency $t$, (MINIMUM REWARDS PER BLOCK EXCLUDING BONUS REWARDS) $t$ denotes the minimum number of coins rewarded for the miners of cryptocurrency $t$, and (TOTAL COINS WHEN FULLY MINED) $t$ denotes the number of total coins of cryptocurrency $t$, given the cryptocurrency is fully mined. 
Table 3. Testing the differences-in-means between functioning and default cryptocurrencies

\begin{tabular}{|c|c|c|c|}
\hline & Default (D) & Functioning (F) & Difference (F-D) \\
\hline Minimum Reward & 65880.65 & 3377.064 & $\begin{array}{l}-62503.6^{* *} \\
(-1.97)\end{array}$ \\
\hline Block time & 160.79 & 152.92 & $\begin{array}{l}7.87 \\
(0.30)\end{array}$ \\
\hline Ret_D1 & 0.0403 & 0.7124 & $\begin{array}{l}0.6721 * * * \\
(3.17)\end{array}$ \\
\hline Ret_W1 & 0.2541 & 0.2849 & $\begin{array}{l}0.0309 \\
(0.19)\end{array}$ \\
\hline Ret_M1 & 0.2454 & 0.1197 & $\begin{array}{l}-0.1257^{* *} \\
(-2.31)\end{array}$ \\
\hline Vol_D1 & 3.1776 & 10.2559 & $\begin{array}{l}7.0783 * * \\
(2.43)\end{array}$ \\
\hline Vol_W1 & 2.7361 & 4.8749 & $\begin{array}{l}2.1388 \\
(0.93)\end{array}$ \\
\hline Vol_M1 & 0.6147 & 0.3131 & $\begin{array}{l}-0.3016^{*} \\
(-1.78)\end{array}$ \\
\hline MTTCR & $3.2 \mathrm{E}-05$ & $8.0 \mathrm{E}-06$ & $\begin{array}{l}-2.4 \mathrm{E}-05 \\
(-1.57)\end{array}$ \\
\hline PMTTCR & 0.0152 & 0.0041 & $\begin{array}{l}-0.0111 * * \\
(-2.47)\end{array}$ \\
\hline Pre-mined & $4.89 \mathrm{E}+07$ & $6.14 \mathrm{E}+08$ & $\begin{array}{l}-5.65 \mathrm{E}+08 * * \\
(-2.03)\end{array}$ \\
\hline Known founder & 0.79 & 0.58 & $\begin{array}{l}-0.21 * * * \\
(-8.62)\end{array}$ \\
\hline Scrypt algorithm & 0.80 & 0.60 & $\begin{array}{l}-0.20^{* * * *} \\
(-8.33)\end{array}$ \\
\hline
\end{tabular}

Note: This table reports the differences of the means of our predictor variable candidates between our sample of functioning cryptocurrencies and those that went into default. As potential predictor variable candidates we consider the minimum reward, block time, first day return (Ret_D1), first week return (Ret_W1), first month return (Ret_M1), first day volatility (Vol_D1), first week volatility (Vol_W1), first month volatility (Vol_M1), Minimum-Reward-to-Total-Coins-Ratio (MTTCR), Pre-Mined-To-Total-Coins-Ratio (PMTTCR), and premined coins (pre-mined). Our data set consists of all cryptocurrencies that incorporated the Proof-of-Work mechanism and started trading prior to December 31, 2014. We followed those cryptocurrencies until the end of 2018. We retrieved 146 cryptocurrencies, of which 86 went into default (D) in the sample period and 60 remained functioning $(F)$. (F-D) measures the mean-difference between the functioning and default sample. The corresponding $t$-statistics are given in parentheses.

*Statistically significant on a $10 \%$ level.

** Statistically significant on a 5\% level.

$* * *$ Statistically significant on a $1 \%$ level.

Next, we employed Multiple Linear Discriminant Analysis (MLDA) to address our research question. MDLA, which is a type of cluster analysis, has been used to model credit risks. For instance, in his seminal paper, Altman (1968) explored bankruptcy among companies in the 
manufacturing industry and proposed the z-score to predict the probability that a firm will go bankrupt within two years. That research led to many modifications being applied to predict various types of financial failure (Altman, 1983; 2002; Altman, Hartzell, and Peck, 1995; Altman, Haldeman, and Narayanan, 1977; Altman, Danovi, and Falini, 2013; Altman, and Rijken, 2010). This is the first paper to make use of MLDA to model defaults in the cryptocurrency market. Again, all input variables used in our model were available to the naïve investor within one month after a cryptocurrency started trading. Since there are different methodologies to perform cluster analysis, below we explain how we set up our model.

We divided the data into two groups, the default group, and the group that consists of functioning cryptocurrencies. We stacked the data of those two groups into two matrices defined as $\boldsymbol{X}_{\mathbf{1}}$ and $\boldsymbol{X}_{2}$, where $\boldsymbol{X}_{\mathbf{1}}$ denotes the default group and $\boldsymbol{X}_{\mathbf{2}}$ denotes the functioning group. Moreover, the matrix $\boldsymbol{X}$ defines the whole data set, that is,

$$
X=\left[\begin{array}{l}
X_{1} \\
X_{2}
\end{array}\right]=\left[\begin{array}{lll}
{\left[x_{1,1}\right.} & \ldots & x_{1, K}
\end{array}\right]
$$

Let us assume that we consider $K$ variables of the cryptocurrency-specific data and let us also assume that we deal with $T_{1}$ cryptocurrencies that went into default and $T_{2}$ cryptocurrencies that were functioning during our sample period. For instance in Equation's (1) notation, $\boldsymbol{x}_{1,1}$ defines a $T_{1} \times 1$ column vector that contains the values for variable 1 for the default sample (e.g., group 1), whereas $x_{1, K}$ defines a $T_{1} \times 1$ column vector that contains the values for variable $K$ in the default sample, and so forth. More concretely,

$$
\boldsymbol{x}_{1,1}=\left[\begin{array}{c}
x_{1,1} \\
x_{2,1} \\
\vdots \\
x_{T_{1}, 1}
\end{array}\right] \text {, or } \boldsymbol{x}_{1, K}=\left[\begin{array}{c}
x_{1, K} \\
x_{2, K} \\
\vdots \\
x_{T_{1}, K}
\end{array}\right] \text {, and analogously } \boldsymbol{x}_{2,1}=\left[\begin{array}{c}
x_{T_{1}, 2} \\
x_{T_{1}+1,2} \\
\vdots \\
x_{T, 2}
\end{array}\right] \text {, or } \boldsymbol{x}_{1, K}=\left[\begin{array}{c}
x_{T_{1}, K} \\
x_{T_{1}+1, K} \\
\vdots \\
x_{T, K}
\end{array}\right] \text {. }
$$

Then for the matrices $\boldsymbol{X}_{\mathbf{1}}$ and $\boldsymbol{X}_{2}$, the sample average of each column can be stacked into the $1 \times K$ vectors $\boldsymbol{\mu}_{1}$ and $\boldsymbol{\mu}_{2}$, given by

$$
\boldsymbol{\mu}_{1}=\left[\begin{array}{lll}
\overline{\boldsymbol{x}}_{1,1} & \ldots & \overline{\boldsymbol{x}}_{1, K}
\end{array}\right] \quad \text { and } \quad \boldsymbol{\mu}_{2}=\left[\begin{array}{lll}
\overline{\boldsymbol{x}}_{2,1} & \ldots & \overline{\boldsymbol{x}}_{2, K}
\end{array}\right]
$$


For instance, the element $\overline{\boldsymbol{x}}_{1,1}=\frac{1}{T_{1}} \sum_{t=1}^{T_{1}} x_{t, 1}$ defines the sample average of the first cryptocurrency-specific variable of the default group and $\bar{x}_{2,1}=\frac{1}{\left(T-T_{1}\right)} \sum_{t=T_{1}+1}^{T} x_{t, 2}$ defines the corresponding sample average of the first cryptocurrency-specific variable of the functioning group. Moreover, the global mean vector $\boldsymbol{\mu}$ stacks the overall sample averages for each column of the matrix $\boldsymbol{X}$ into a $1 \times K$ row vector. Note that $\boldsymbol{\mu}$ can be simply calculated as

$\boldsymbol{\mu}=\frac{1}{T}\left(T_{1} \boldsymbol{\mu}_{1}+\left(1-T_{1}\right) \boldsymbol{\mu}_{2}\right)=\frac{1}{T}\left(T_{1} \boldsymbol{\mu}_{1}+T_{2} \boldsymbol{\mu}_{2}\right) \equiv\left[\begin{array}{llll}\mu_{1} & \mu_{2} & \cdots & \mu_{K}\end{array}\right]$

Then we calculated the mean-corrected matrices $\boldsymbol{X}_{\mathbf{1}}^{\mathbf{0}}$ and $\boldsymbol{X}_{\mathbf{2}}^{\mathbf{0}}$ defined as

$\boldsymbol{X}_{\mathbf{1}}^{\mathbf{0}}=\left[\begin{array}{c}\boldsymbol{x}_{1,1}-\boldsymbol{\mu} \\ \boldsymbol{x}_{2,1}-\boldsymbol{\mu} \\ \vdots \\ \boldsymbol{x}_{T_{1}, 1}-\boldsymbol{\mu}\end{array}\right] \quad$ and $\quad \boldsymbol{X}_{\mathbf{2}}^{\mathbf{0}}=\left[\begin{array}{c}\boldsymbol{x}_{T_{1}+1,2}-\boldsymbol{\mu} \\ \boldsymbol{x}_{T_{1}+2,2}-\boldsymbol{\mu} \\ \vdots \\ \boldsymbol{x}_{T, 2}-\boldsymbol{\mu}\end{array}\right]$

where obviously $T-T_{1}=T_{2}$ and given Equation's (4) notation, $\boldsymbol{x}_{t, i}-\boldsymbol{\mu}$ defines a $1 \times K$ row vector $i$ in each respective matrix, $\boldsymbol{X}_{\mathbf{1}}$ and $\boldsymbol{X}_{2}$, subtracted by the global mean vector $\boldsymbol{\mu}$. For instance,

$\boldsymbol{x}_{1,1}-\boldsymbol{\mu}=\left[\begin{array}{llll}\left(x_{1,1}-\mu_{1}\right) & \left(x_{1,2}-\mu_{2}\right) & \ldots & \left(x_{1, K}-\mu_{K}\right)\end{array}\right]$, or

$\boldsymbol{x}_{2,1}-\boldsymbol{\mu}=\left[\begin{array}{lllll}\left(x_{2,1}-\mu_{1}\right) & \left(x_{2,2}-\mu_{2}\right) & \ldots & \left(x_{2, K}-\mu_{K}\right)\end{array}\right]$, for the default group and analogously,

$\boldsymbol{x}_{T_{1}+1,2}-\boldsymbol{\mu}=\left[\begin{array}{llll}\left(x_{T_{1}+1,1}-\mu_{1}\right) & \left(x_{T_{1}+1,2}-\mu_{2}\right) & \ldots & \left(x_{T_{1}+1, K}-\mu_{K}\right)\end{array}\right]$, or

$\boldsymbol{x}_{T_{1}+2,2}-\boldsymbol{\mu}=\left[\begin{array}{llll}\left(x_{T_{1}+2,1}-\mu_{1}\right) & \left(x_{T_{1}+2,2}-\mu_{2}\right) & \ldots & \left(x_{T_{1}+2, K}-\mu_{K}\right)\end{array}\right]$, for the functioning group respectively.

We compounded the corresponding empirical sample covariance matrices as

$C_{1}=\frac{X_{1}^{0^{T}} X_{1}^{0}}{T_{1}} \quad$ and $\quad C_{2}=\frac{X_{2}^{0^{T}} X_{2}^{0}}{T_{2}}$ 
where the dimension of $\boldsymbol{C}_{\mathbf{1}}$ and $\boldsymbol{C}_{\mathbf{2}}$ must be the same, that is, $K \times K$ as we want to investigate the characteristic-specific differences in cryptocurrencies. Then we employed the estimated sample covariance matrices $\boldsymbol{C}_{\mathbf{1}}$ and $\boldsymbol{C}_{\mathbf{2}}$ to calculate the pooled within-group covariance matrix, simply defined as $\boldsymbol{C}(r, s)$ and given by

$\boldsymbol{C}(r, s)=\frac{1}{\left(T_{1}+T_{2}\right)} \sum_{i \in(1,2)} T_{i} \cdot \boldsymbol{C}_{\boldsymbol{i}}(r, s)$,

where $r=1, \ldots, K$ and $s=1, \ldots, K$. As $\operatorname{rank}(\boldsymbol{C})=K$ was satisfied, we then compounded the inverse of $\boldsymbol{C}$, defined as $\boldsymbol{C}^{-1}$. Moreover, the prior-probability vector, based on the empirical data, can simply be calculated as

$\boldsymbol{P}=\left[\begin{array}{l}p_{1} \\ p_{2}\end{array}\right]=\left[\begin{array}{l}\left(\frac{T_{1}}{\left(T_{1}+T_{2}\right)}\right) \\ \left(\frac{T_{2}}{\left(T_{1}+T_{2}\right)}\right)\end{array}\right]$

Finally, for our $T$ cryptocurrencies, we can estimate the discriminant function depending on the default and non-default cluster as

$f_{1, t}=\boldsymbol{\mu}_{1} \boldsymbol{C}^{-\mathbf{1}} \boldsymbol{x}_{\boldsymbol{t}, \boldsymbol{K}}^{T}-0.5 \cdot \boldsymbol{\mu}_{1} \boldsymbol{C}^{-\mathbf{1}} \boldsymbol{\mu}_{1}^{T}+\ln \left(p_{1}\right), \quad$ and

$f_{2, t}=\boldsymbol{\mu}_{2} \boldsymbol{C}^{-\mathbf{1}} \boldsymbol{x}_{\boldsymbol{t}, \boldsymbol{K}}^{T}-0.5 \cdot \boldsymbol{\mu}_{2} \boldsymbol{C}^{-\mathbf{1}} \boldsymbol{\mu}_{2}^{T}+\ln \left(p_{2}\right)$,

where $\boldsymbol{x}_{t, K}^{T}$ is the corresponding transposed $1 \times K$ vector of characteristics of cryptocurrency $t$. If $f_{1, t}>f_{2, t}$, cryptocurrency $t$ is predicted to be in the default group, otherwise it is predicted to be in the functioning group. Given the subsamples, we defined $f_{1, t}>f_{2, t}$ as success for the default group and $f_{1, t}<f_{2, t}$ as success for the functioning group meaning that the discriminant model correctly assigned the respective cryptocurrency to its corresponding group. Furthermore, for each group, we coded two vectors of dummy variables denoted as $\boldsymbol{d}_{1}$ and $\boldsymbol{d}_{2}$ that have a value of one in case of success and a value of zero otherwise. The prediction accuracy of predicting a cryptocurrency's default within four years is then simply given by $\sum_{t=1}^{T_{1}} d_{1, t} / T_{1}$, whereas the Type-I error of this model is $1-\sum_{t=1}^{T_{1}} d_{1, t} / T_{1}$. In the same 
manner, the prediction accuracy for predicting a cryptocurrency's continued functioning can be calculated as $\sum_{t=1}^{T_{2}} d_{2, t} / T_{2}$, while the Type-II error is then given by $1-\sum_{t=1}^{T_{2}} d_{2, t} / T_{2}$.

Setting up the empirical model is ultimately an empirical question. After our initial analysis of differences in sample means, we decided to employ the following cardinal variables in our discriminant analysis: Day-1 return, Month-1 return, and the corresponding volatilities, PMTTCR, and minimum reward. We also account for a set of dummy variables for measuring the qualitative variables algorithm, anonymous founder, and pre-mined. Specifically, we employ $K=9$ predictor variables in our analysis. Since we have $T_{1}=86$ cryptocurrencies that ended up in default and $T_{2}=60$ that remained functioning, our matrices $\boldsymbol{X}_{\mathbf{1}}$ and $\boldsymbol{X}_{\mathbf{2}}$ have the dimension $86 \times 12$ and 60x12, respectively. Our model operates with 12 instead of nine columns because we employ three dummy variables for indicating the algorithm (scrypt, SHA, or others), one binary dummy variable for indicating whether a cryptocurrency is pre-mined, one dummy variable indicting whether the founder is anonymous, and continuous variables for Day-1 return, Day-1 volatility, Month-1 return, Month-1 volatility, actual amount of pre-mined coins, actual amount of minimum reward, and PMTTCR. The estimated discriminant function is reported in Table A.4. and A.5. in the appendix. Finally, these estimates are used to calculate the results reported in Table 4. For example, from Table A.4. we learn that the discriminant function correctly predicts in 75 out of 86 cryptocurrencies that they are in group 1 because the value of the discriminant function is larger for group 1 than for group 2. Consequently, $87.21 \%$ of cryptocurrency defaults are predicted correctly.

Table 4. Predicting cryptocurrency default

\begin{tabular}{lcc}
\hline & Predicted group by the Multiple Linear Discriminant Function \\
\cline { 2 - 3 } Actual Group & Default group & Functioning group \\
Default group & $87.21 \%$ & $12.79 \%$ \\
& & \\
Functioning group & $43.33 \%$ & $56.67 \%$ \\
\hline
\end{tabular}

Note: This table reports the results of our multiple linear discriminant analysis. Our dataset consists of all cryptocurrencies that incorporated the Proof-of-Work mechanism and started trading between 2009 and the end of 2014. We followed up those cryptocurrencies until the end of 2018 . We retrieved 146 cryptocurrencies, of which 86 went into default in the sample period and 60 remained functioning. Our model incorporates the following predictor variables: minimum reward, pre-mined, Day-1 return, Month-1 return, Day-1 volatility, 
Month-1 volatility, and PMTTCR. Moreover, we include a set of dummy variables for indicating 'algorithm' and 'founder anonymity'.

Given the data of bankrupt and functioning cryptocurrencies, as reported in Table A.6. that are in either the default group or the functioning group our model is able to correctly predict $87 \%$ of the defaults corresponding to a Type-I error of $13 \%$. Our estimates are close to models that predict bankruptcy of enterprises. For instance, the popular multiple discriminant model from Altman (1968) predicted 94\% of bankruptcies of U.S. firms in the manufacturing industry. It is important to note, however, that first Altman's (1968) benchmark model uses recent information on those companies investigated because he employed data from balance sheets that were released about one year before the bankruptcy occurred. Second, he matched that sample of companies that went bankrupt with a sample of matched companies having the same number of firms and the same firm characteristics, whereas our analysis accounts for the whole sample of available cryptocurrencies. Furthermore, we use only information available at an early stage, that is, after one month of trading. Its chosen sample means our model predicts bankruptcy within the next four years, which is very different from Altman's findings. Even though Altman's (1968) model performed remarkably well for a one- and two-year period prior to bankruptcy, a robustness check shows that its success rate is only $29 \%$ for a four year period. ${ }^{12}$ Even though our results suggest that our cryptocurrency default prediction model is an accurate forecaster of failure, Table 4 shows that the Type-II error is $43 \%$. This result implies that our model struggles to predict functioning cryptocurrencies.

\subsection{Robustness checks}

Since we only have one sample available, our estimates reported in Table 4 are only point estimates. To investigate how sensitive our model is with respect to resampling and to compound confidence intervals for our estimates, we employed bootstrapping. It seems reasonable to assume that characteristic $k$ of cryptocurrency $t$ is uncorrelated with the characteristic $k$ of cryptocurrency $s$, that is, $\operatorname{cov}\left(x_{t, k}, x_{s, k}\right)=0 .{ }^{13}$ However, characteristic $k$ of cryptocurrency $t$ is not necessarily uncorrelated with characteristic $l$, meaning $\operatorname{cov}\left(x_{t, k}, x_{t, l}\right) \neq 0$. We have ensured this is ex-ante by simply choosing our research set-up because all cryptocurrencies have the same consensus protocol and are therefore

\footnotetext{
${ }^{12}$ The average success rate of Altman's (1968) model between year one and four prior to bankruptcy is $61 \%$.

${ }^{13}$ Note that Altman $(1968$, p.592) highlights that "there is reason to believe that some of the measurements will have a high degree of correlation or collinearity with each other."
} 
homogenous. However, characteristics of a cryptocurrency could be - at least potentially correlated with other characteristics of the same cryptocurrency. To account for this issue, we employed a pairs bootstrap as detailed by Godfrey (2009, pp.183-185). In doing so, we constructed new data matrices defined as $\boldsymbol{X}_{1}^{b}$ and $\boldsymbol{X}_{2}^{b}$ where each row vector in $\boldsymbol{X}_{\mathbf{1}}$ and $\boldsymbol{X}_{\mathbf{2}}$ is randomly resampled with replacement where each row in $\boldsymbol{X}_{\mathbf{1}}$ and $\boldsymbol{X}_{\mathbf{2}}$ is drawn with probability $1 / T_{1}$ and $1 / T_{2}$ respectively. We employ $B=5000$ bootstrap samples and reestimate the corresponding discriminant functions to estimate the empirical confidence interval.

More concretely, for each bootstrap run $b$, we employ the original data matrix $\boldsymbol{X}_{\mathbf{1}}$ that has the dimension $86 \times 12$, as described in section 2.1 . Then we randomly draw with replacement and with probability $1 / 86=0.0116$ a row from matrix $\boldsymbol{X}_{\mathbf{1}}$ and add that row into matrix $\boldsymbol{X}_{1}^{b}$ to construct a new data matrix. For each run $b$, this procedure is stopped when all 86 rows in the new data matrix $\boldsymbol{X}_{1}^{b}$ are filled. In the same manner, for each bootstrap run $b$, we employ the original data matrix $\boldsymbol{X}_{2}$ that has the dimension $60 \times 12$, as described in section 2.1. Then we randomly draw with replacement and with probability $1 / 60=0.0167$ a row from matrix $\boldsymbol{X}_{2}$ and add that row into matrix $\boldsymbol{X}_{2}^{b}$ to construct a new data matrix. For each run $b$, this procedure is stopped when all 60 rows in the new data matrix $\boldsymbol{X}_{2}^{b}$ are filled. These new data matrices are used to run the linear discriminant analysis described in section 2.1 for each bootstrap iteration $b=1, \ldots, 5000$. The corresponding point estimates are stored in vector. These vectors are sorted in an increasing order. Then, the 125th observation gives us the value of the lower bound and the 4875th observation gives us the upper bound of our confidence interval covering $95 \%$ probability.

The results of our analysis can be found in Table 5. Using bootstrapping, the $95 \%$ confidence interval for our point estimate concerning successfully predicting cryptocurrency default is between $83.72 \%$ and $89.53 \%$ again suggesting a high level of accuracy. However, the $95 \%$ confidence interval for the Type-II error ranges between $41.67 \%$ and $53.33 \%$. Assuming that the point estimate for the Type-II error is distributed as $N\left(\mu_{1}, \sigma\right)$ with $\mu_{1}=$ 47.50 and $\sigma=2.97$, and that the corresponding point estimate for the correctly predicted functioning cryptocurrencies is distributed as $N\left(\mu_{2}, \sigma\right)$ with $\mu_{2}=52.50,60.99 \%$ of the confidence intervals are overlapping. ${ }^{14}$ This result implies that our model overpredicts defaults in the sample of functioning cryptocurrencies.

${ }^{14}$ Note, $\mu_{1}=\frac{(41.67+53.33)}{2}=47.50, \sigma=\frac{(53.33-47.50)}{1.96}=\frac{(58.33-52.50)}{1.96}=2.9745, \mu_{2}=\frac{(46.67+5.33)}{2}=52.50$. 
Table 5. Estimated confidence intervals using bootstrapping

\begin{tabular}{lcc}
\hline & Predicted group by the Multiple Linear Discriminant Function \\
\cline { 2 - 3 } Actual Group & Default group & Functioning group \\
Default group & {$[83.72 \% ; 89.53 \%]$} & {$[10.47 \% ; 16.28 \%]$} \\
& & \\
Functioning group & {$[41.67 \% ; 53.33 \%]$} & {$[46.67 \% ; 58.33 \%]$} \\
\hline
\end{tabular}

Note: This table reports the results of $B=5000$ bootstrap replications using a pairs bootstrap. We constructed new data matrices by random resampling with replacement using a probability of $1 / T_{1}$ for the subsample of default cryptocurrencies and a probability of $1 / T_{2}$ for the subsample of functioning cryptocurrencies. Then we re-estimated our model $B$ times and sorted the estimated probabilities in an increasing order. The $125^{\text {th }}$ observation gives us the value of the lower bound and the $4875^{\text {th }}$ observation gives us the upper bound of our confidence interval covering $95 \%$ probability.

It is important to note that the new digital financial markets evolve fast over time. For instance, during 2016 few cryptocurrencies were launched implementing SHA algorithms. ${ }^{15}$ Moreover, we would like to stress that new cryptocurrencies are applying more advanced algorithms and security mechanisms; there are only few new cryptocurrencies implementing the SHA hashing algorithms and PoW mechanism because these methods have slower speeds and higher energy consumption. Moreover, as technology advances so too does the blockchain. The most common algorithms for the cryptocurrencies issued before 2015 were SHA and Scrypt, but new cryptocurrency algorithms like X11-X17 were created specifically for Graphical Processing Unit (GPU) mining and provide good profit levels to the Portable Instant Mining Platform (PiMP) community since the rise of large Application-Specific Integrated Circuits (ASICs) for Scrypt. Table A.7. in the appendix provides a brief overview of those new algorithms X11-X17. New research needs to account for technological changes associated with cryptocurrencies.

In the same way like Altman (1968) proposed in his seminal paper a model that had the ability to predict bankruptcies for firms in a specific industry in the U.S. (e.g., manufacturing industry), our paper takes the first step in exploring predictable patterns in defaults in new emerging digital financial markets. Expecting that we could propose a universal model being capable of predicting defaults across different categories of digital

\footnotetext{
${ }^{15}$ See footnote 8 .
} 
asset would be an illusion. As pointed out in Howell, Niessner and Yermack (2019) there are three types of digital assets.

While cryptocurrencies that are defined as "general-purpose medium of exchange and store of value cryptocurrency" can be considered as alternative to traditional fiat currency, utility tokens or security tokens have a very different purpose, which is financing business projects. Due to their nature, those digital assets have very different characteristics compared to cryptocurrencies. While our paper specifically governs cryptocurrencies that incorporate the PoW consensus protocol - which obviously was the dominant consensus protocol in our sample of investigation - future research is needed to explore the predictability of defaults for either cryptocurrencies that follow other consensus protocols (e.g., Proof-of-Stake or Hybrid), or other types of digital currencies, such as tokens issued in Initial Coin Offerings.

Moreover, the research methodology of our paper is related to the literature applying MLDA to predict various types of financial failure (e.g., Altman, 1968; Altman, 1983; 2002; Altman, Hartzell, and Peck, 1995; Altman, Haldeman, and Narayanan, 1977; Altman, Danovi, and Falini, 2013; Altman, and Rijken, 2010). However, there are other strands of literature dealing with analyzing credit risks and employ methodologies such as Probit/Logit models. Future research is encouraged to investigate the predictability of defaults using other methodologies than MLDA also.

\section{Conclusion}

In this age of digital finance, investors can now choose from more than 2,000 cryptocurrencies to invest in. Among cryptocurrencies that started trading prior to December 2014, we found $59 \%$ went bankrupt by the end of 2018. This paper proposes a model to predict cryptocurrency default. We downloaded data for all cryptocurrencies launched between January 2009 and December 2014 and established if they went bankrupt by December 2018. We explored almost two dozen cryptocurrency-specific variable candidates that might serve as predictor variables. From those variables, we only used data for the model setup available to the naïve investor one month after a new cryptocurrency started trading. For each of the selected variables, we estimated the sample means for both the default sample and the functioning sample. Our model correctly predicts 75 of 86 bankruptcies $(87 \%)$. Employing bootstrapping established that the estimates are statistically significant. Notably, the new digital asset markets are subject to technological changes: For instance, many 
cryptocurrencies issued after 2015 adopted the PoS (minting) consensus mechanism due to greater energy consumption of PoW (mining). Other major changes that we identified are among others that new cryptocurrencies adopt more efficient and profitable algorithms like $\mathrm{X} 11, \mathrm{X} 12$, and X17 over previously popular algorithms like SHA and Scrypt. Therefore, future research on such technological changes is warranted. Nevertheless, our proposed model could be employed in the asset management industry, for instance, as a screening tool for investment decision making. A rational investor would avoid investing in digital assets exhibiting overly high default risks. Portfolios that pre-condition the set of digital assets on those cryptocurrencies that are not predicted as at risk of bankruptcy might generate a better risk-return profile for investors. 


\section{References}

Altman, E., 1968. Financial Ratios, Discriminant Analysis and the Prediction of Corporate Bankruptcy. Journal of Finance 23, 189-209.

Altman, E. I., Haldeman, R.G. and Narayanan, P. 1977. ZETA Analysis: A New Model to Identify Bankruptcy Risk of Corporations. Journal of Banking and Finance 129-54.

Altman, E. 2000, Predicting Financial Distress of Companies: Revisiting the Z-score and Zeta Models, New York University Working Paper.

Altman, E.1983. Corporate Financial Distress. New York: Wiley Interscience.

Altman E., 2002. Revisiting Credit Scoring Models in a Basel 2 Environment. Salomon Center for the Study of Financial Institutions 2, 2-37.

Altman, E., Hartzell, J. and Peck, M., 1995. Emerging Markets Corporate Bonds: A Scoring System. New York: Wiley and Sons.

Altman, E.I, Danovi, A. and Falini, A., 2013. Z-Score Models' Application to Italian Companies Subject to Extraordinary Administration. Journal of Applied Finance 23, 128-137.

Altman, E.I. and Rijken, H., 2010. Assessing Sovereign Debt Default Risk: A Bottom-up Approach. Journal of Applied Corporate Finance 41, 1-30.

Altman, E.I., 2018. Applications of Distress Prediction Models: What Have We Learnt After 50 Years from the Z-Score Models? International Journal of Financial Studies 6, 1-15.

Ardia, D., K. Bluteau, and M. Rüede, 2018. Regime changes in Bitcoin GARCH volatility dynamics, Finance Research Letters 29, 266-271.

Baur, D.G., and T. Dimpfl, 2018. Asymmetric volatility in cryptocurrencies. Economics Letters 173, 148-151.

Borri, N., 2019. Conditional tail-risk in cryptocurrency markets. Journal of Empirical Finance 50, 1-19.

Catania, L., Grassi, S. and F. Ravazzolo, 2019. Forecasting cryptocurrencies under model and parameter instability. International Journal of Forecasting 35, 485-501.

Chaim, P., and M.P. Laurini, 2019. Is Bitcoin a bubble? Physica A: Statistical Mechanics and its Applications 517, 222-232.

Cheah, E.T. and Fry, J., 2015. Speculative bubbles in Bitcoin markets? An empirical investigation into the fundamental value of Bitcoin. Economics Letters 130, 32-36.

Dwyer, G.P., 2015. The economics of Bitcoin and similar private digital currencies. Journal of Financial Stability 17, 81-91. 
Dyhrberg, A.H., 2016. Hedging capabilities of Bitcoin. Is it the virtual gold?. Finance Research Letters 16, 139-144.

Fry, J. and Cheah, E.T., 2016. Negative bubbles and shocks in Cryptocurrency markets. International Review of Financial Analysis 47, 343-352.

Godfrey, L., 2009. Bootstrap Test for Regression Models. Palgrave MacMillan, New York.

Howell, S.T., Niessner, M., and Yermack, D. 2019. Initial Coin Offerings: Financing Growth with Cryptocurreny Token Sales. Working paper, Leonard N. Stern School of Business.

Katsiampa, P., 2017. Volatility estimation for Bitcoin: A comparison of GARCH models. Economics Letters 158, 3-6.

Kethineni, S., and Y. Cao, 2019. The Rise in Popularity of Cryptocurrency and Associated Criminal Activity. International Criminal Justice Review, forthcoming.

Lahmiri, S., and S. Bekiros, 2019. Cryptocurrency forecasting with deep learning chaotic neural networks. Chaos, Solutions and Fractals 118, 35-40.

Li, J. and Rahgozar, R., 2012. Application of the Z -Score Model with Consideration of Total Assets Volatility in Predicting Corporate Financial Failures from 2000-2010. Journal of Accounting and Finance 12, 11-19.

Li, Z.-Z., Tao, R., Su, C.-W., and O.-R. Lobont, 2019. Does Bitcoin bubble burst? Quality and Quantity 53, 91-105.

Maume, P., 2019. Initial Coin Offerings and EU Prospectus Disclosure. European Business Law Review, forthcoming.

Omane-Adjepong, M., Alagidede, P., and N.K. Akosah, 2019. Wavelet time-scale persistence analysis of cryptocurrency market returns and volatility. Physica A: Statistical Mechanics and its Applications 514, 105-120.

Osterrieder, J. and Lorenz, J., 2017. A statistical risk assessment of Bitcoin and its extreme tail behavior. Annals of Financial Economics 12, 1750003.

Satish, Y.M. and B. Janakiram, 2011. Turnaround Strategy Using Altman Model as a Tool in Solar Water Heater Industry in Karnataka. International Journal of Business and Management 6, 199-206.

Snedecor, G. W. and Cochran, W. G. (1989). Statistical Methods. Eighth Edition, Iowa State University Press.

Shen, D., Urquhart, A., and P. Wang, 2019. Does twitter predict Bitcoin? Economics Letters $174,118-122$.

Urquhart, A., 2016. The inefficiency of Bitcoin. Economics Letters 148, 80-82. 


\section{Appendix}

Table A.1. Cryptocurrency characteristics

\begin{tabular}{ll}
\hline Category & Details \\
\hline Resource and history & Website, announcement, whitepaper, block explorer, github, etc. \\
Coin specifications & $\begin{array}{l}\text { Coin name, type, founder(s), contributor(s), block time, security } \\
\text { mechanism, algorithm, staking maturity, block size, launch type, etc. }\end{array}$ \\
$\begin{array}{l}\text { Daily trading, } \\
\text { supply and distribution }\end{array}$ & $\begin{array}{l}\text { rank, market cap, price (\$), price (BTC), volume(24h), market } \\
\text { Dominance (Volume, Value), Supply (Max, Total, Circulating), etc. }\end{array}$ \\
Economics & Block reward, inflation, fees recipient, funding model, etc. \\
Privacy & $\begin{array}{l}\text { Cryptographic privacy, sender privacy, recipient privacy, hidden } \\
\text { transaction amount, transaction link broken, balances visible, } \\
\text { anonymous holdings, network trust required, quantum-proof privacy, } \\
\text { trusted setup, auditable supply, mobile privacy, etc. }\end{array}$
\end{tabular}

Features and scaling

Instant send, protocol level, governance, voters, multi-signature support, scaling model, transparent transaction size (bytes), private transaction size (bytes), most throughput in a block, prunable blockchain, etc.

Wallets

Ledger, trezor, coinomi, jaxx, native mobile wallet binaries for all major OS, webwallet, etc.

Network and masternodes Largest miner or pool, entities controlling, staking supply, public nodes, masternodes, masternode cost (coin), masternode cost (\$), etc.

Community Percentage of active users, number of subscribers, facebook likes, Twitter followers, Alexa rank, Google/Bing searches, etc.

Note: This table provides an overview of different features and characteristics of a cryptocurrency. (Source: https://news.bitcoin.com) 
Table A.2. Potential cryptocurrency-specific variables for the model

\section{Potential categorical variable candidates}

\begin{tabular}{ll}
\hline 1.Security mechanism & PoW/PoS/Hybrid/Others \\
2.Launch type* & Standard/ICO/Fork/Coinswap/others \\
3.Algorithms & SHA/Scrypt/others \\
4.Funding model* & ICO/donations/founders/others \\
\hline
\end{tabular}

\section{Potential Binary Variable Candidates}

5. Pre-mined, 6.Privacy choice*, 7.Sender privacy*, 8.Recipient

Privacy*, 9.Network trust required*, 10. Multi-signature

YES/NO

Support*, 11.Founder anonymity

\section{Potential Continuous Variable Candidates}

12. Block time, 13.Block reward, 14.Block size*, 15.Pre-mined ratio, 16.Total coins, 17. Volume*, 18.Return, 19.Volatility, 20. Reward percentage

Note: This table provides an overview of different quantifiable (continuous/categorical/binary) cryptocurrencyspecific variables that could potentially be used to develop a model. Our model incorporates 9 variables from the 20 candidates. The remaining variables were excluded owing to the non-accessibility of websites for many dead coins highlighted with an asterisk $(*)$. 
Table A.3. Descriptive statistics of functioning and bankrupt cryptocurrencies

Panel A. Descriptive statistics of the functioning sample

\begin{tabular}{|c|c|c|c|c|c|c|c|c|c|}
\hline & $\mathbf{N}$ & Mean & Median & Maximum & Minimum & Std. Dev. & Skewness & Kurtosis & Jarque-Bera \\
\hline PMTTCR & 60 & 0.0041 & 0.0000 & 0.0842 & 0.0000 & 0.0153 & 4.5660 & 23.2754 & 1236.2090 \\
\hline MTTCR & 60 & 0.0000 & 0.0000 & 0.0004 & 0.0000 & 0.0000 & 7.3888 & 56.3109 & 7651.0780 \\
\hline Ret_D1 & 60 & 0.7124 & -0.3779 & 18.3576 & -0.9655 & 3.1486 & 3.7353 & 18.8502 & 767.6010 \\
\hline Ret_W1 & 60 & 0.2849 & -0.0753 & 16.2296 & -0.8501 & 2.2079 & 6.4717 & 46.9459 & 5246.9390 \\
\hline Ret_M1 & 60 & 0.1197 & 0.0000 & 2.6973 & -0.7500 & 0.5512 & 2.6563 & 11.9317 & 269.9996 \\
\hline Vol_D1 & 60 & 10.2559 & 0.4855 & 337.0018 & 0.0000 & 45.5486 & 6.4127 & 45.7924 & 4989.2090 \\
\hline Vol_W1 & 60 & 4.8749 & 0.0694 & 263.4009 & 0.0000 & 33.9713 & 7.5306 & 57.8118 & 8077.9110 \\
\hline Vol_M1 & 60 & 0.3131 & 0.0116 & 7.2756 & 0.0000 & 1.1050 & 5.0058 & 29.2786 & 1976.9990 \\
\hline Block time & 60 & 152.9167 & 60.0000 & 600.0000 & 5.0000 & 185.9005 & 1.7929 & 4.6947 & 39.3247 \\
\hline Minimum reward & 60 & 3377.0640 & 50.0000 & 100000.0000 & 0.0000 & 14473.7900 & 5.6653 & 36.1092 & 3061.5140 \\
\hline Pre-mined & 60 & $6.1400 \mathrm{E}+08$ & $0.0000 \mathrm{E}+00$ & $3.6800 \mathrm{E}+10$ & $0.0000 \mathrm{E}+00$ & $4.7500 \mathrm{E}+09$ & $7.5509 \mathrm{E}+00$ & $5.8017 \mathrm{E}+01$ & $8.1373 E+03$ \\
\hline \multirow[t]{2}{*}{ Total coins } & 60 & $2.9500 \mathrm{E}+10$ & $8.4000 \mathrm{E}+07$ & $5.0000 \mathrm{E}+11$ & $4.2000 \mathrm{E}+01$ & $1.0000 \mathrm{E}+11$ & $3.8711 \mathrm{E}+00$ & $1.6909 \mathrm{E}+01$ & $6.3351 \mathrm{E}+02$ \\
\hline & \multicolumn{9}{|c|}{ Panel B. Descriptive statistics of the default sample } \\
\hline PMTTCR & 86 & 0.0152 & 0.0000 & 0.5000 & 0.0000 & 0.0754 & 6.1213 & 39.2322 & 5241.1920 \\
\hline MTTCR & 86 & 0.0000 & 0.0000 & 0.0024 & 0.0000 & 0.0003 & 9.0733 & 83.5495 & 24429.4600 \\
\hline Ret_D1 & 86 & 0.0403 & -0.4245 & 13.4928 & -0.9815 & 1.7926 & 5.4962 & 39.2686 & 5146.5500 \\
\hline Ret_W1 & 86 & 0.2541 & -0.0911 & 13.4928 & -0.9827 & 1.6441 & 6.3551 & 50.4816 & 8657.5260 \\
\hline Ret_M1 & 86 & 0.2454 & 0.0405 & 4.6154 & -0.4512 & 0.7490 & 3.7423 & 18.5781 & 1070.3270 \\
\hline Vol_D1 & 86 & 3.1776 & 0.3235 & 182.0544 & 0.0000 & 19.8910 & 8.6488 & 77.9195 & 21185.1800 \\
\hline Vol_W1 & 86 & 2.7361 & 0.1414 & 182.0544 & 0.0000 & 19.6609 & 8.9798 & 82.3686 & 23728.5600 \\
\hline Vol_M1 & 86 & 0.6147 & 0.0152 & 21.3018 & 0.0000 & 2.6716 & 6.1461 & 44.6106 & 6745.7830 \\
\hline Block time & 86 & 160.7907 & 60.0000 & 3600.0000 & 10.0000 & 407.9158 & 7.2223 & 60.3602 & 12537.5000 \\
\hline Minimum reward & 86 & 65880.6500 & 50.0000 & 5000000.0000 & 0.2500 & 541325.8000 & 8.9668 & 82.1671 & 23610.7400 \\
\hline Pre-mined & 86 & $4.8800 \mathrm{E}+07$ & $0.0000 \mathrm{E}+00$ & $1.8200 \mathrm{E}+09$ & $0.0000 \mathrm{E}+00$ & $2.3400 \mathrm{E}+08$ & $6.1705 \mathrm{E}+00$ & $4.3285 \mathrm{E}+01$ & $6.3612 \mathrm{E}+03$ \\
\hline Total coins & 86 & $1.4000 \mathrm{E}+10$ & $1.0000 \mathrm{E}+08$ & $5.5000 \mathrm{E}+11$ & $3.2000 \mathrm{E}+04$ & $6.3300 \mathrm{E}+10$ & $7.3333 \mathrm{E}+00$ & $6.1404 \mathrm{E}+01$ & $1.2994 \mathrm{E}+04$ \\
\hline
\end{tabular}

Note: This table reports the descriptive statistics for the following 12 variables: Pre-Mined-To-Total-Coin-Ratio (PMTTCR), Minimum-Reward-To-Total-Coin-Ratio (MTTCR), first day return (Ret D1), first week return (Ret W1), first month return (Ret M1), first day volatility (Vol D1), first week volatility (Vol W1), first month volatility (Vol M1), block time (in seconds), minimum reward for the miners per block (minimum reward), number of coins mined before issued to the public (pre-mined), and the total number of coins of the Cryptocurrency (total coins). The figures are reported for both, the running sample (Panel A) and the default sample (Panel B). 
Table A.4. Discriminant function for the default group

\begin{tabular}{|c|c|c|c|c|c|}
\hline $\mathbf{t}$ & $\begin{array}{l}\text { Predicted } \\
\text { group } 1\end{array}$ & $\begin{array}{c}\text { Predicted } \\
\text { group } 2\end{array}$ & $\mathbf{t}$ & $\begin{array}{l}\text { Predicted } \\
\text { group } 1\end{array}$ & $\begin{array}{l}\text { Predicted } \\
\text { group } 2\end{array}$ \\
\hline 1 & 27.3805 & 27.7258 & 44 & 24.5862 & 23.9807 \\
\hline 2 & 25.7955 & 26.5482 & 45 & 25.1247 & 24.3461 \\
\hline 3 & 29.1404 & 28.5218 & 46 & 23.6011 & 24.0290 \\
\hline 4 & 28.5050 & 28.3511 & 47 & 23.7185 & 24.0802 \\
\hline 5 & 28.4949 & 28.3009 & 48 & 26.6271 & 27.7649 \\
\hline 6 & 26.7095 & 25.0682 & 49 & 23.6858 & 24.0478 \\
\hline 7 & 29.0677 & 29.6769 & 50 & 22.7344 & 22.5435 \\
\hline 8 & 27.3892 & 27.7573 & 51 & 24.1190 & 23.7822 \\
\hline 9 & 28.7611 & 28.5356 & 52 & 24.6971 & 24.2462 \\
\hline 10 & 23.3757 & 22.8601 & 53 & 25.1351 & 24.5761 \\
\hline 11 & 26.2717 & 23.9860 & 54 & 24.8930 & 24.4421 \\
\hline 12 & -24.1924 & -24.2417 & 55 & 23.4671 & 23.1464 \\
\hline 13 & 24.5396 & 23.8052 & 56 & 25.5403 & 24.3222 \\
\hline 14 & 25.0503 & 24.3044 & 57 & 25.0424 & 23.9827 \\
\hline 15 & 25.2780 & 24.4149 & 58 & 27.0499 & 25.4708 \\
\hline 16 & 28.3193 & 28.2433 & 59 & 29.1556 & 28.5972 \\
\hline 17 & 28.7954 & 28.5569 & 60 & 25.4615 & 24.2150 \\
\hline 18 & 27.1257 & 25.3986 & 61 & 25.7609 & 24.4991 \\
\hline 19 & 27.4460 & 25.7223 & 62 & 25.6308 & 24.3650 \\
\hline 20 & 24.9086 & 23.8277 & 63 & 25.5393 & 24.4207 \\
\hline 21 & 28.8249 & 28.2669 & 64 & 24.8019 & 23.8088 \\
\hline 22 & 24.3948 & 23.4981 & 65 & 26.0950 & 24.7936 \\
\hline 23 & 25.5785 & 24.3492 & 66 & 24.2334 & 23.3495 \\
\hline 24 & 24.5392 & 23.7033 & 67 & 25.4390 & 24.2220 \\
\hline 25 & 25.5527 & 24.3702 & 68 & 25.3581 & 24.1425 \\
\hline 26 & 25.2765 & 24.4344 & 69 & 25.9102 & 24.6305 \\
\hline 27 & 25.1694 & 24.2734 & 70 & 26.1701 & 24.7816 \\
\hline 28 & 27.1309 & 24.7269 & 71 & 23.4963 & 23.2327 \\
\hline 29 & 25.4649 & 24.5927 & 72 & 25.0475 & 24.2988 \\
\hline 30 & 25.4501 & 24.6327 & 73 & 24.6484 & 23.9325 \\
\hline 31 & 24.4508 & 24.0105 & 74 & 25.4414 & 24.5399 \\
\hline 32 & 26.4005 & 25.3303 & 75 & 25.3188 & 24.4492 \\
\hline 33 & 25.0495 & 24.2478 & 76 & 26.1223 & 25.0992 \\
\hline 34 & 25.7881 & 24.8164 & 77 & 24.1029 & 25.0230 \\
\hline 35 & 24.8941 & 24.1895 & 78 & 24.9693 & 24.2222 \\
\hline 36 & 28.7836 & 28.5683 & 79 & 25.9494 & 24.9470 \\
\hline 37 & 27.7893 & 27.7997 & 80 & 24.8711 & 24.0899 \\
\hline 38 & 25.4256 & 24.4933 & 81 & 25.8052 & 24.8806 \\
\hline 39 & 24.8828 & 24.1603 & 82 & 23.0776 & 22.8346 \\
\hline 40 & 23.6977 & 23.4344 & 83 & 24.9735 & 23.4067 \\
\hline 41 & 28.5788 & 28.3744 & 84 & 24.4830 & 23.9345 \\
\hline 42 & 28.5522 & 28.3965 & 85 & 24.4737 & 23.8478 \\
\hline 43 & 28.6334 & 28.1262 & 86 & 24.1142 & 24.5685 \\
\hline
\end{tabular}

Note: This table reports the values for the discriminant function (Equation 8.a) for the default group (e.g., group 1). 
Table A.5. Discriminant function for the functioning group

\begin{tabular}{|c|c|c|c|c|c|}
\hline $\mathbf{t}$ & $\begin{array}{c}\text { Predicted } \\
\text { group } 1\end{array}$ & $\begin{array}{c}\text { Predicted } \\
\text { group } 2\end{array}$ & t & $\begin{array}{c}\text { Predicted } \\
\text { group } 1\end{array}$ & $\begin{array}{c}\text { Predicted } \\
\text { group } 2\end{array}$ \\
\hline 1 & 27.82065 & 27.73309 & 31 & 23.81871 & 23.66742 \\
\hline 2 & 28.21115 & 28.06119 & 32 & 22.69601 & 22.69963 \\
\hline 3 & 27.56446 & 27.8617 & 33 & 25.47647 & 24.56926 \\
\hline 4 & 24.88217 & 26.79222 & 34 & 26.46251 & 27.41852 \\
\hline 5 & 24.18907 & 23.8235 & 35 & 24.05865 & 24.03279 \\
\hline 6 & 27.64701 & 27.89729 & 36 & 23.55639 & 23.60259 \\
\hline 7 & 28.47438 & 28.74628 & 37 & 26.2968 & 27.56041 \\
\hline 8 & 28.23995 & 28.78224 & 38 & 26.63752 & 27.78143 \\
\hline 9 & 28.43512 & 28.02157 & 39 & 23.94774 & 24.32457 \\
\hline 10 & 27.85306 & 27.72618 & 40 & 27.08734 & 28.17422 \\
\hline 11 & 24.93163 & 23.89347 & 41 & 26.38169 & 27.58451 \\
\hline 12 & 25.24417 & 24.02586 & 42 & 26.65863 & 27.79309 \\
\hline 13 & 24.07879 & 23.36516 & 43 & 26.46865 & 27.67717 \\
\hline 14 & 25.39226 & 24.0771 & 44 & 21.97226 & 22.86871 \\
\hline 15 & 25.19025 & 24.36664 & 45 & 28.81073 & 29.50451 \\
\hline 16 & 31.00211 & 30.156 & 46 & 24.47789 & 24.03402 \\
\hline 17 & 24.91486 & 24.19066 & 47 & 24.29782 & 25.81669 \\
\hline 18 & 25.57118 & 24.62532 & 48 & 25.75796 & 24.53365 \\
\hline 19 & 26.27598 & 25.23614 & 49 & 26.17337 & 25.1366 \\
\hline 20 & 25.00121 & 24.35303 & 50 & 28.75249 & 28.56327 \\
\hline 21 & 28.64485 & 28.4349 & 51 & 24.91487 & 24.19067 \\
\hline 22 & 26.96299 & 27.15382 & 52 & 25.01501 & 24.15877 \\
\hline 23 & 24.91487 & 24.19067 & 53 & 25.19886 & 24.33645 \\
\hline 24 & 24.4346 & 23.66766 & 54 & 24.09113 & 24.42255 \\
\hline 25 & 26.47026 & 26.85933 & 55 & 23.3386 & 23.74375 \\
\hline 26 & 26.01675 & 25.00639 & 56 & -23.2155 & -22.1288 \\
\hline 27 & 22.73395 & 23.34834 & 57 & 28.3670 & 28.27678 \\
\hline 28 & 24.55047 & 23.99539 & 58 & 24.27292 & 23.87617 \\
\hline 29 & 25.37536 & 24.50556 & 59 & 25.17099 & 24.26115 \\
\hline 30 & 22.85169 & 23.04122 & 60 & -22.6525 & -21.7619 \\
\hline
\end{tabular}

Note: This table reports the values for the discriminant function (Equation 8.b) for the functioning group (e.g., group 2). 
Table A.6. Name and symbol of cryptocurrencies used for the study

Panel A. Name and symbol of running cryptocurrencies

\begin{tabular}{|c|c|c|c|c|c|c|c|c|c|c|c|c|c|c|}
\hline S.No. & Cryptocurrency & Symbol & S.No. & Cryptocurrency & Symbol & S.No. & Cryptocurrency & Symbol & S.No. & Cryptocurrency & Symbol & S.No. & Cryptocurrency & Symbol \\
\hline 1 & Blakecoin & BLC & 13 & Fedoracoin & TIPS & 25 & Reddcoin & RDD & 37 & Bitcoin & BTC & 49 & SmartCoin & SMC \\
\hline 2 & Maxcoin & MAX & 14 & Novacoin & NVC & 26 & NobleCoin & NOBL & 38 & Peercoin & PPC & 50 & Gridcoin & GRC \\
\hline 3 & Zurcoin & ZUR & 15 & Spots & SPT & 27 & Mooncoin & MOON & 39 & Zetacoin & ZET & 51 & Lucky7Coin & LK7 \\
\hline 4 & DimeCoin & DIME & 16 & Diamond & DMD & 28 & Phoenixcoin & PXC & 40 & Unobtanium & UNO & 52 & 42 coin & $42 \mathrm{C}$ \\
\hline 5 & Quark & QRK & 17 & Royalcoin & RYC & 29 & Fastcoin & FST & 41 & Bytecoin & $\mathrm{BCN}$ & 53 & Goldcoin & GLD \\
\hline 6 & Animecoin & ANI & 18 & Worldcoin & WDC & 30 & Argentum & ARG & 42 & Terracoin & TRC & 54 & Huntercoin & HUC \\
\hline 7 & Primecoin & XPM & 19 & Mincoin & MNC & 31 & Florincoin & FLO & 43 & Namecoin & NMC & 55 & Curecoin & CURE \\
\hline 8 & Vertcoin & VTC & 20 & Megacoin & MEC & 32 & Annoncoin & ANC & 44 & Tekcoin & TEK & 56 & Stellar & XLM \\
\hline 9 & Litecoin & LTC & 21 & Feathercoin & FTC & 33 & Grandcoin & GDC & 45 & Skeincoin & SKC & 57 & Trollcoin & TROLL \\
\hline 10 & Bullion & $\mathrm{CBX}$ & 22 & Dogecoin & DOGE & 34 & Supercoin & SUPER & 46 & CDNcoin & $\mathrm{CDN}$ & 58 & SecureCoin & SRC \\
\hline 11 & Communitycoin & COMM & 23 & Galaxycoin & GLX & 35 & Betacoin & BET & 47 & Bela Coin & BELA & 59 & Marscoin & MARS \\
\hline 12 & Emerald & EMD & 24 & BitBar & ВTB & 36 & iXcoin & IXC & 48 & Redcoin & RED & 60 & Pandacoin & PND \\
\hline \multicolumn{15}{|c|}{ Panel B. Name and symbol of default cryptocurrencies } \\
\hline S.No. & Cryptocurrency & Symbol & S.No. & Cryptocurrency & Symbol & S.No. & Cryptocurrency & Symbol & S.No. & Cryptocurrency & Symbol & S.No. & Cryptocurrency & Symbol \\
\hline 1 & Datacoin & DTC & 18 & Sexcoin & SXC & 35 & Doubloons & DBL & 52 & Metiscoin & MTS & 69 & Cagecoin & CAGE \\
\hline 2 & Tagcoin & TAG & 19 & Xivra & XIV & 36 & CHNCoin & $\mathrm{CNC}$ & 53 & Unioncoin & UNC & 70 & Electric & VOLT \\
\hline 3 & Nyancoin & NYAN & 20 & Extremecoin & EXC & 37 & Globalcoin & GLC & 54 & Frozencoin & FZ & 71 & Bottlecaps & CAP \\
\hline 4 & Paycoin & XPY & 21 & Americancoin & AMC & 38 & Krugercoin & KGC & 55 & KingdomCoin & KING & 72 & Neocoin & NEC \\
\hline 5 & Infinitecoin & IFC & 22 & Lottocoin & LOTTO & 39 & Franko & FRK & 56 & Memecoin & MEM & 73 & Bitgem & BTG \\
\hline 6 & Qubitcoin & Q2C & 23 & Graincoin & GRA & 40 & Netcoin & NET & 57 & Solcoin & SOL & 74 & Lebowskis & LBW \\
\hline 7 & Freicoin & FRC & 24 & Xencoin & $\mathrm{XNC}$ & 41 & BBQcoin & $\mathrm{BQC}$ & 58 & Hypercoin & HYC & 75 & Growthcoin & GRW \\
\hline 8 & AllAgesCoin & AAC & 25 & Batcoin & BAT & 42 & Catcoin & CAT & 59 & Craftcoin & $\mathrm{CRC}$ & 76 & Prospercoin & PRC \\
\hline 9 & Joincoin & $\mathrm{J}$ & 26 & Junkcoin & JKC & 43 & Memorycoin & MMC & 60 & Nanotoken & NAN & 77 & Hobbitcoin & $\mathrm{HBC}$ \\
\hline 10 & $\begin{array}{l}\text { Cthulhu } \\
\text { Offerings }\end{array}$ & OFF & 27 & StarCoin & STR & 44 & Luckycoin & LKY & 61 & GIL & GIL & 78 & Hotcoin & HTC \\
\hline 11 & Colossuscoin & $\mathrm{COL}$ & 28 & Zenithcoin & ZTC & 45 & Yacoin & YAC & 62 & Usecoin & USE & 79 & Lovecoin & LOVE \\
\hline 12 & Particle & PRT & 29 & HoboNickels & HBN & 46 & AsicCoin & ASC & 63 & AlphaCoin & ALF & 80 & Bells & BEL \\
\hline 13 & Pennies & CENT & 30 & Philosopherstone & PHS & 47 & Tigercoin & TGC & 64 & Richcoin & $\mathrm{RCH}$ & 81 & Zeuscoin & ZEU \\
\hline 14 & Qqcoin & QQC & 31 & CACHeCoin & $\mathrm{CACH}$ & 48 & Devcoin & DVC & 65 & Zedcoin & ZED & 82 & ELACoin & ELC \\
\hline 15 & Applecoin & $\mathrm{APC}$ & 32 & Microcoin & MRC & 49 & Teacoin & TEA & 66 & Stablecoin & $\mathrm{SBC}$ & 83 & EzCoin & EZC \\
\hline 16 & ZcCoin & $\mathrm{ZCC}$ & 33 & Kittehcoin & MEOW & 50 & Copperlark & CLR & 67 & Socialcoin & SOC & 84 & Noirbits & NRB \\
\hline \multirow[t]{2}{*}{17} & Onecoin & ONE & 34 & Gamecoin & GME & 51 & Chaincoin & $\mathrm{CHC}$ & 68 & ElephantCoin & ELP & 85 & Nibble & NBL \\
\hline & & & & & & & & & & & & 86 & Globe & GLB \\
\hline
\end{tabular}

Note: This table reports the name and symbol of cryptocurrencies with PoW consensus protocol issued prior to the end of year 2014. These cryptocurrencies were tracked till the end of year 2018 and categorized them into running (Panel A) and default (Panel B) samples. 
Table A.7. Algorithms X11. X13. X14. X15. and X17

\begin{tabular}{|c|c|c|c|c|c|c|c|c|c|c|c|c|c|c|c|c|}
\hline 1 & 2 & 3 & 4 & 5 & 6 & 7 & 8 & 9 & 10 & 11 & 12 & 13 & 14 & 15 & 16 & 17 \\
\hline Blake & BMW & Groestl & $\mathrm{JH}$ & Keccak & Skein & Luffa & Cubehash & Shavite & SIMD & Echo & & & & & & \\
\hline \multicolumn{11}{|c|}{$\mathrm{X} 11$} & Hamsi & Fugue & & & & \\
\hline \multicolumn{13}{|c|}{$\mathrm{X} 13$} & Shabal & & & \\
\hline \multicolumn{14}{|c|}{ X14 } & Whirlpool & & \\
\hline \multicolumn{15}{|c|}{$\mathrm{X} 15$} & Loselose & Djb2 \\
\hline
\end{tabular}

Note: This table shows the chain of different hashing algorithms X11. X13. X14. X15. and X17 with their sub-algorithms (Source: getpimp.org) 